\title{
LA ENSEÑANZA DEL INGLÉS COMO LENGUA EXTRANJERA EN EL BACHILLERATO: PROPUESTA CURRICULAR CENTRADA EN EL DESARROLLO DE HABILIDADES LINGÜÍSTICAS BÁSICAS
}

\author{
Mario A. Castillo Sánchez Hidalgo
}

\section{RESUMEN}

Es una verdad innegable: la enseñanza del inglés ocupa, hoy, gran parte de los afanes educativos en distintas áreas del sistema educativo mexicano.

Como lengua extranjera, la enseñanza del inglés ha sufrido, en los diversos ámbitos educativos y en la mayoría de los casos, modificaciones tendientes a lograr el desarrollo comunicativo de los estudiantes a partir de las habilidades lingüísticas básicas.

El tópico central de este trabajo es la enseñanza del inglés como lengua extranjera en el Bachillerato. La globalización obliga a responder a esta demandante realidad a través de la adecuación, instrumentación y modernización de las estructuras que permitan, en términos generales, volver más competente a México. La educación juega -y jugará- un papel crucial para las generaciones presentes y futuras, como medio de perfeccionamiento de las capacidades humanas y desempeñará una función integradora estructural que precisa desarrollarse ante el nuevo orden mundial.

En el ámbito educativo, el Bachillerato representa la última oportunidad del docente para incidir realmente en los estudiantes, formarlos y ayudarlos a acometer la compleja tarea de proseguir su preparación profesional, o bien, insertarse al mercado laboral. 


\section{A. CONSIDERACIONES GENERALES SOBRE EL BACHILLERATO}

En nuestro país, el Bachillerato cuenta con diversos subsistemas y modalidades: por una parte está el universitario al que pertenecen los dos Bachilleratos impartidos por la Universidad Nacional Autónoma de México, la Escuela Nacional Preparatoria y el Colegio de Ciencias y Humanidades, más los 24 Bachilleratos dependientes de diversas universidades estatales: los subsistemas de Bachillerato, coordinados por la Secretaría de Educación Pública (Colegios de Bachilleres, Escuelas Preparatorias Incorporadas, Preparatorias Federales por Cooperación y Centros de Estudio del Bachillerato ${ }^{1}$ ).

Existen, además, los Bachilleratos estatales sujetos a las Secretarías de Educación de los Estados; el Bachillerato de Arte supeditado al Instituto Nacional de Bellas Artes; y el Bachillerato Militar que depende de la Secretaría de la Defensa Nacional.

\section{A.1. EL BACHILLERATO UNIVERSITARIO}

Entre los Bachilleratos universitarios destacan: la Escuela Nacional Preparatoria y el Colegio de Ciencias y Humanidades. La Escuela Nacional Preparatoria cuenta con nueve planteles. Incluye un conjunto de materias comunes, obligatorias para todos los alumnos y una serie de materias específicas organizadas por áreas.

El Colegio de Ciencias y Humanidades fue creado el 26 de enero de 1971, bajo el rectorado del doctor Pablo González Casanova. Sus objetivos son:

1. La utilización óptima de los recursos destinados a la educación.

2. La formación sistemática e institucional de nuevos cuadros de enseñanza media superior.

${ }^{1}$ MEDINA, Sara Rosa (1996). Educación y modernidad: el Bachillerato en México ante los desafíos del Tercer Milenio. México: Universidad Autónoma de San Luis Potosí. 
3. Un tipo de educación que constituya un ciclo en sí mismo, que puede ser preparatorio, terminal y profesional a un nivel que no requiere aún la licenciatura.

El plan de estudios del $\mathrm{CCH}$ se orienta a facilitar que los estudiantes aprendan cómo se aprende; está diseñado de manera que los tres primeros semestres hacen énfasis en el método experimental y la sociedad, así como las formalizaciones del lenguaje español y las matemáticas. El cuarto semestre insiste en la síntesis racional (matemáticas y método científico experimental, teoría de la historia, ensayos de investigación y análisis de la expresión escrita). El quinto y sexto, subrayan la comprobación del dominio de los métodos del conocimiento y su aplicación a campos específicos de la ciencia, buscando la formación universal, la orientación profesional y la capacitación propedéutica.

\section{A.1.1. BACHILLERATOS DE LAS UNIVERSIDADES}

Éstos forman orgánicamente parte de las mismas; por ello, están sujetos a sus condiciones y circunstancias de trabajo. Manifiestan una gran variedad de posiciones curriculares, que dan lugar a planes y programas de estudio diferentes, aun cuando su objetivo primordial sea el mismo: preparar para estudios superiores.

\section{A.1.2. EL BACHILLERATO COORDINADO POR LA SECRETARÍA DE EDUCACIÓN PÚBLICA}

El órgano normativo que regula el Bachillerato coordinado por la Secretaría de Educación Pública ha venido cambiando su denominación:

-1980, Subdirección de Educación Media Superior y Orientación.

- 1981, Dirección General de Educación Media Superior.

- 1989, Unidad de Educación Media Superior.

- 1992, Dirección General del Bachillerato (su actual denominación). 
Esta dirección coordina los subsistemas de los Colegios de Bachilleres, las Escuelas Preparatorias Particulares Incorporadas ${ }^{2}$, las Preparatorias Federales por Cooperación, los Centros de Estudios del Bachillerato, el Sistema del Bachillerato Semiescolarizado, el Sistema de Bachillerato Abierto y la Escuela Federal Lázaro Cárdenas.

En 1992, de conformidad con el Programa para la Modernización Educativa se inició la revisión curricular del plan de estudios, entonces vigente para los subsistemas coordinados por la Dirección General del Bachillerato. En 1993 se puso en práctica, como plan piloto, el denominado Currículum Marco en los colegios de bachilleres estatales, las escuelas preparatorias particulares incorporadas y las preparatorias federales por cooperación.

Durante 1994 se realizaron el análisis y la valoración de esta propuesta curricular, así como su reestructuración, originando el denominado Currículum Básico Nacional, que es el vigente.

\section{A.1.3. ESCUELAS PREPARATORIAS PARTICULARES INCORPORADAS}

Estas Escuelas, incorporadas a la Secretaría de Educación Pública, conforman un subsistema regido por los mismos criterios y la normatividad general del Bachillerato dependiente de esta Secretaría.

Dentro del marco del Programa para la Modernización Educativa 1989-1994, se implementó, en el ciclo escolar 1993-1994, un nuevo plan de estudios producto de una consulta en los distintos sectores sociales. A un año de su operación, se ajustó a un mapa curricular llamado Currículum Básico Nacional, bajo cuyos lineamientos operará en lo sucesivo este subsistema.

Dentro de él existen seis Escuelas Preparatorias Incorporadas mediante acuerdo secretarial, con autonomía en la elaboración de su plan y programas de estudio, previo dictamen

Al año de 1996, existían 244 Escuelas Preparatorias Particulares Incorporadas, con 48031 alumnos. Cfr. Medina. Op.cit. p.55. 
académico de la Secretaría de Educación Pública a través de la Dirección General del Bachillerato. Estas escuelas son las preparatorias de las siguientes instituciones educativas: la Universidad Autónoma de Guadalajara, la Universidad del Valle de México, la Universidad Anáhuac, la Universidad Tecnológica de México, la Escuela Bancaria y Comercial y los CETYS.

Son escuelas preparatorias particulares incorporadas a la Secretaría de Educación Pública las que

- Reúnen las características señaladas en las fracciones I y II del Artículo $1^{\circ}$.

- Dependen, en su organización y administración internas, de una persona particular, física o moral, de conformidad con lo dispuesto por la Ley Federal de Educación y demás ordenamientos aplicables de la Secretaría de Educación Pública.

- Han sido reconocidas, oficialmente, como «escuelas preparatorias particulares incorporadas a la Secretaría de Educación Pública" y se les ha otorgado la clave de registro correspondiente.

Las finalidades que estas escuelas preparatorias se proponen alcanzar son las indicadas en el Artículo 5ำ de la Ley General de Educación:

1. Promover el desarrollo armónico de la personalidad, para que se ejerzan en plenitud las capacidades humanas.

2. Crear y fortalecer la conciencia de la nacionalidad y el sentido de la convivencia internacional.

3. Alcanzar, mediante la enseñanza de la lengua nacional, un idioma común para todos los mexicanos, sin menoscabo del uso de las lenguas autóctonas.

4. Proteger y acrecentar los bienes y valores que constituyen el acervo cultural de la nación y hacerlos accesibles a la colectividad.

${ }^{3}$ SECRETARÍA DE EDUCACIÓN PÚBliCA (1988). Mamual de Normas Generales para las Escuelas Preparatorias Federales por Cooperación y Particulares Incorporadas a la Secretaría de Educación Pública.

Dirección General de Educación Media Superior. Subdirección de Operación. 
5. Fomentar el crecimiento y el respeto a las instituciones nacionales.

6. Enriquecer la cultura con impulso creador y con la incorporación de ideas y valores universales.

7. Hacer conciencia de la necesidad de un mejor aprovechamiento social de los recursos naturales y contribuir a preservar el equilibrio ecológico.

8. Promover las condiciones sociales que lleven a la distribución equitativa de los bienes materiales y culturales, dentro de un régimen de libertad.

9. Formar conciencia sobre la necesidad de una planeación familiar con respeto a la dignidad humana y sin menoscabo de la libertad.

10. Vigorizar los hábitos intelectuales que permitan el análisis objetivo de la realidad.

11. Propiciar las condiciones indispensables para el impulso de la investigación, la creación artística y la difusión de la cultura.

12. Lograr que las experiencias y los conocimientos obtenidos al adquirir, transmitir y acrecentar la cultura, se integren de tal modo que se armonicen tradición e innovación.

13. Fomentar y orientar la actividad científica y tecnológica de manera que responda a las necesidades del desarrollo nacional independiente.

14. Promover las actitudes solidarias para el logro de una vida social justa.

15. Enaltecer los derechos individuales y sociales y postular la paz universal, basada en el reconocimiento de los derechos económicos, políticos y sociales de las naciones ${ }^{4}$.

\section{B. DESARROLLO DE COMPETENCIAS ACADÉMICAS BÁSICAS}

Considerado este marco normativo, el estudiante desarrollará además las competencias académicas básicas (CAB) que

${ }^{4}$ SEP. Op. cit. p. 60 
precisa ante los retos que la educación media superior posee actualmente (propios, nacionales y mundiales).

El concepto competencias académicas básicas (CAB) ha adquirido gran importancia porque la mayoría de los países están en proceso de revisar y modificar sus sistemas educativos ${ }^{5}$.

La tendencia más difundida es mantener a los jóvenes en la escuela con el fin de proporcionarles una mejor formación. México ha entrado en un proceso de modernización a partir de 1988. Sin embargo, se manifiesta un fenómeno contradictorio: las demandas sociales de personal altamente calificado han aumentado y la calidad académica se ha abatido.

En Estados Unidos se iniciaron estudios e investigaciones, destacando el conducido por la GAO (General Accounting Office $)^{6}$. Abocados a preparar a la juventud para el mercado de empleo, se han definido una serie de competencias, certificadas a partir de ciertos estándares nacionales, para lograr una adecuada transición de la escuela al mundo del trabajo.

Hasta épocas recientes, el concepto competencia aludía sólo al nivel superior. Actualmente es de uso común para aplicarse a los estudios correspondientes a la educación media superior.

El término competencia se define de diversas formas. Lerner -citado por Medina- establece que la sociedad moderna demanda el dominio verbal, escrito y matemático de parte de sus trabajadores? ${ }^{7}$ La demanda de trabajo calificado es alta.

La noción de competencia apunta al desempeño individual: uno es capaz o incapaz de lograr un objetivo, ejecutar una tarea y ser evaluado a través de la aplicación de un criterio de medida. La excelencia está reservada a una minoría, la competencia no. Ésta puede definirse como la capacidad de un individuo para realizar una tarea o como un nivel de ejecución

\footnotetext{
${ }^{5}$ Medina. Ibídem.

${ }^{6}$ GAO (General Accounting Office) (1990). Training Strategies preparing Noncollege Youth for Employment in USA and foreign Countries. Report to Congressional Requesters. Washington: GAO.

${ }^{7}$ Medina. Ibidem.
} 
o dominio requerido para desempeñarse adecuadamente en la sociedad. De esta forma, las competencias son indicadores de una exitosa ejecución o dominio de las actividades cotidianas.

Para los académicos del College Board de los Estados Unidos, el concepto competencia envuelve un conjunto de objetivos y metas relativas a la misión del sistema educativo en lo general, y de las instituciones que lo conforman, en lo particular.

El Bachillerato debe dotar al alumno de competencias que puedan evaluarse a partir de una serie de estándares consensados.

Los adolescentes pueden alcanzar una competencia si están capacitados para ejecutar una tarea determinada:

1. Al elegir un método de enseñanza que asegure el éxito.

2. Una aproximación diagnóstica.

3. Una acción remedial.

Britell — citado por Medina-define competencia como el conocimiento de una materia o de una habilidad reconocida. Competencia es un estado de ejecución adecuado a una tarea.

Las CAB destacan la capacidad de comunicación oral y escrita, la posibilidad de identificar información relevante acerca de un problema, su interpretación y clasificación en forma útil; buscan la relación entre la información nueva y la adquirida previamente.

Las competencias académicas son aquellas habilidades amplias, esenciales para el trabajo efectivo en todos los campos de los estudios académicos. Proporcionan el vínculo que articula todas las disciplinas. Estas competencias son:

- Lectura.

- Capacidad para expresarse por escrito y oralmente.

- Facultad para escuchar.

- Capacidad de observación.

- Matemáticas entendidas como lenguaje.

- Capacidad de estudio.

- Capacidad para razonar.

- Desarrollo de una cultura informática.

${ }^{8}$ Ibídem. 


\section{EL ENFOQUE COMUNICATIVO Y SU VINCULACIÓN CON LAS COMPETENCIAS ACADÉMICAS BÁSICAS}

En el ámbito de la enseñanza de lenguas (sobre todo extranjeras), el Enfoque Comunicativo se ha destacado como la metodología dominante. El Enfoque Comunicativo establece que una lengua deberá estudiarse desde las necesidades prácticas que tiene (a esas necesidades las llama funciones). A fin de que la lengua pueda desarrollarse organizadamente, el alumno fomentará habilidades lingüísticas concretas (hablar, escuchar, leer y escribir). Se trata, en términos generales, de que el estudiante no olvide su dimensión de hablante de una lengua. Las habilidades —entendidas como operaciones concretas para comunicarse- pueden, efectivamente, entenderse como competencias comunicativas.

El origen del Enfoque Comunicativo se remonta a los cambios ocurridos en la tradición británica sobre la enseñanza de lenguas (finales de los 60). En Estados Unidos, el Enfoque Comunicativo surge como resultado de los severos enjuiciamientos que hiciera Noam Chomsky en su texto Syntactic Structures, Estructuras sintácticas.

Este autor había demostrado que las teorías estructuralistas de la lengua eran incapaces de considerar sus características fundamentales: creatividad y naturaleza irrepetible del habla, entre otras. Además, los lingüistas británicos enfatizaron otro aspecto que había soslayado el Método Audiolingual: el aspecto funcional y comunicativo de la lengua ${ }^{9}$. Tanto británicos como norteamericanos observaron la necesidad de enfocar la enseñanza de la lengua a partir de la competencia comunicativa, más que en la destreza para el manejo de ciertas estructuras gramaticales.

$\mathrm{Al}$ respecto, es importante señalar las valiosas aportaciones teóricas de Candlin y Widdowson para otorgar, al Enfoque Comunicativo, una sólida fundamentación lingüística.

${ }^{9}$ Es importante recordar que el Método Audiolingual constituyó durante algún tiempo la metodología dominante en la enseñanza de lenguas extranjeras. 
Además, el entorno había cambiado notablemente, sobre todo en Europa. Con la creciente interdependencia entre naciones, surgió la necesidad de realizar importantes esfuerzos para que los adultos aprendieran las principales lenguas del naciente Mercado Común Europeo.

En 1971, un grupo de expertos investigó la posibilidad de desarrollar cursos de lenguas extranjeras en un sistema basado en unidades, cada una articulada de manera autónoma a partir del desarrollo de ciertas habilidades, sobre todo comunicativas. Cada unidad o "porción", guardaría una consecuente relación tomando en cuenta las necesidades estudiantiles.

El lingüista británico D. A. Wilkins propuso una definición funcional o comunicativa de la lengua, que sirviera de base para el desarrollo de un currículum comunicativo en la enseñanza de lenguas ${ }^{10}$.

Pretendía demostrar los sistemas de significados que subyacen en los usos comunicativos de la lengua. Describió dos tipos de significados: categorías nocionales (con conceptos como tiempo, secuencia, cantidad, locación, frecuencia) y categorías funcionales, empleadas en la comunicación cotidiana (favores, rechazos, ofrecimientos, quejas). De hecho el libro de Wilkins, Notional Syllabuses, influyó considerablemente tanto en el desarrollo del Enfoque Comunicativo, como en el diseño de programas y de materiales de instrucción en Europa.

Diversos factores y autores contribuyeron a que rápidamente el Enfoque Comunicativo gozara de amplia aceptación. Destacan los trabajos del Consejo Europeo, los de lingüistas como Wilkins, Widdowson, Candlin, Brumfit, Johnson, entre otros; además de que estos postulados teóricos fueron aplicados en materiales para la enseñanza de lenguas extranjeras.

Los postulados del Enfoque Comunicativo podrían sintetizarse en:

a) El objetivo que deberá alcanzarse en la enseñanza de lenguas extranjeras es el desarrollo de la "competencia comunicativa".

${ }^{10}$ WILKINS, D. A. (1976). Notional Syllabuses. Oxford: Oxford University Press. 
b) Un curso de lenguas bajo este enfoque, buscará el desarrollo de las cuatro habilidades lingüísticas (producción oral, producción escrita, comprensión de lectura y comprensión auditiva) que reconozcan la interdependencia entre lengua y comunicación.

En un documento titulado Syllabuses for Primary Schools, Jack Richards concibió la enseñanza comunicativa bajo el principio de que, cuando menos, existen dos partes involucradas en un proceso de interacción o de transacción de algo. Una parte posee una intención de comunicar algo y la otra reacciona a esa intención ${ }^{11}$.

Por otro lado, Howatt, manifestó dos versiones del Enfoque Comunicativo. En primer término consideró que esta metodología enfatiza la importancia de dotar a los estudiantes de las oportunidades para el uso del inglés para propósitos comunicativos y pretende integrar estas oportunidades hacia una concepción amplia de la enseñanza de lenguas. Posteriormente, mencionó que el lenguaje es adquirido mediante la comunicación, y que el docente deberá estimular el desarrollo de la capacidad del lenguaje que los alumnos tienen. Concluyó señalando que bajo el Enfoque Comunicativo, los estudiantes usan el inglés para aprenderlo (using the English to learn $i t)^{12}$.

\section{EL DISEÑO CURRICULAR PARA LA ENSEÑNANZA DEL INGLÉS}

Se describe ahora un modelo que, desde la realidad del Bachillerato, y con el Enfoque Comunicativo como sustento teórico, posibilite el desarrollo de habilidades lingüísticas básicas en la enseñanza del inglés como lengua extranjera ${ }^{13}$.

\footnotetext{
${ }^{11}$ RICHARDS, Jack. (1985). The Context of Language Teaching. Cambridge: Cambridge University Press.

${ }^{12}$ HOWATT, A. P. R (1984). A History of English Language Teaching. Oxford: Oxford University Press.

${ }^{13}$ En el apéndice se presenta un cuadro integrador de los elementos centrales de esta propuesta.
} 


\section{D.1. ALGUNAS PRECISIONES SOBRE LA PROPUESTA}

Antes de diseñar el currículum centrado en habilidades lingüísticas básicas para la enseñanza del inglés en el Bachillerato, es importante señalar algunos aspectos que permitan hacer necesaria su existencia. Para ello se expone lo siguiente:

\section{D.1.1. CORRESPONDENCIA DE OBJETIVOS}

Tanto el Bachillerato, centrado en Competencias Académicas Básicas, como el currículum centrado en el desarrollo de habilidades lingüísticas primarias para la enseñanza del inglés en el Bachillerato, comparten sustancialmente los mismos objetivos.

El currículum propuesto encuentra plena articulación dentro de este contexto general, al desarrollar habilidades que, sumadas, permitan alcanzar un grado de competencia académica.

El contexto general de modernización que vive el país establece la necesidad de dotar a los estudiantes de una mejor capacidad de comunicación. El inglés como lengua extranjera se ha ido convirtiendo en una herramienta necesaria para la comunicación global y para la vida laboral. En ese sentido, la propuesta curricular de desarrollo de habilidades lingüísticas encuentra amplia justificación ante la realidad mexicana.

En el ámbito de las competencias académicas vinculadas con la lectura, se enumeran aquellas que guardan estrecha relación con la enseñanza del inglés:

- Capacidad para expresarse por escrito y oralmente.

- Facultad para escuchar.

Es indudable la asociación entre las competencias académicas vinculadas a la lectura con la enseñanza del inglés a partir del desarrollo de habilidades lingüísticas primarias. El Enfoque Comunicativo reconoce, en la lectura, una habilidad que debe desarrollarse a fin de que el estudiante interactúe eficazmente en diferentes contextos comunicativos. 


\section{D.1.2. NIVELES DEL DISEÑO CURRICULAR}

A partir de una propuesta que recoge las posturas de César Coll ${ }^{14}$ y Jack Richards, se enumeran los principales niveles que el diseño curricular para la enseñanza del inglés en el Bachillerato debe considerar:

\section{D.1.2.1. Objetivos del Método de Desarrollo de Habilidades Lingüísticas}

El planteamiento curricular nace desde los postulados teóricos del Communicative Approach (Enfoque Comunicativo) hacia un método que desarrolle las habilidades lingüísticas primarias (hablar, escuchar, leer, escribir). Es decir, desde la lengua entendida a partir de su uso en situaciones comunicativas hacia las habilidades.

El objetivo del método es, entonces, el desarrollo de habilidades lingüísticas primarias.

\section{D.1.2.2. Selección de Contenidos}

Jack Richards establece que el Enfoque Comunicativo seleccionará tópicos de interés comunicativo para los estudiantes ${ }^{15}$. El criterio de selección tomará en cuenta situaciones comunicativas que deriven en funciones gramaticales y en el consecuente desarrollo de habilidades lingüísticas. Aunque la tarea no es sencilla, se pretende seleccionar aquellos tópicos o situaciones comunicativas de carácter general y en concordancia con los intereses de los estudiantes del Bachillerato.

\section{D.1.2.3. Tareas de Aprendizaje y Actividades de Enseñanza}

Parten de la necesidad de desarrollar las habilidades lingüísticas pertinentes a cada uno de los tópicos o contenidos comunicativos.

${ }^{14}$ COLL, César (1999). Psicología y currículum: una aproximación psicopedagógica a la elaboración del currículum escolar. México: Paidós.

${ }^{15}$ Richards. Op. cit. pp.14 y ss. 


\section{D.1.2.4. Rol de los Estudiantes}

El método propuesto recoge las intenciones de César Coll: construir un currículum lo suficientemente personalizado hacia las necesidades de los estudiantes. El énfasis es el aprendizaje de los alumnos, concebidos como sujetos activos y conscientes de sus propios procesos de aprendizaje.

\section{D.1.2.5. Rol de los Profesores}

El profesor es, más bien, un coordinador que facilitará la tarea de los estudiantes hacia la construcción de su aprendizaje. Será tarea del profesor proveer y crear situaciones comunicativas que posibiliten la interacción de los estudiantes al interior del aula. Sin embargo, una buena parte del curso dependerá del estudio autónomo que realicen éstos, por lo que el rol del docente es monitorear y evaluar los aprendizajes.

\section{D.1.2.6. Rol de los Materiales Didácticos}

Los materiales que compongan un curso interesado en desarrollar habilidades lingüísticas primarias para la enseñanza del inglés deberán ser, en primer término, relevantes.

Por relevancia se entiende la importancia que tengan en la construcción de las habilidades comunicativas de los estudiantes. Cada vez que sea posible, se recurrirá a materiales auténticos ${ }^{16}$.

La elaboración de materiales por parte de los docentes es altamente aconsejable, toda vez que sirvan para reflejar puntualmente las necesidades de los estudiantes ${ }^{17}$.

\footnotetext{
${ }^{16}$ Por materiales auténticos se entienden periódicos, revistas, entrevistas, programas de radio o de televisión. Se trata de ofrecer a los alumnos elementos reales de la lengua en situaciones reales.

${ }^{17}$ Jack Richards señala que en el diseño de materiales dentro del Enfoque Comunicativo, es importante que éstos se centren en habilidades comunicativas de expresión, negociación e interpretación; se centren en intercambios de información relevante, interesante y fácil de entender más que en la sola presentación de formas gramaticales; se ofrezcan en diversos tipos de formas (artículos, cassettes, fotografías, etcétera).
} 


\section{D.1.3. COMPONENTES CURRICULARES}

En el modelo enunciado, tres son los elementos centrales de la propuesta curricular (tópicos; funciones gramaticales; habilidades lingüísticas) además de los mecanismos de evaluación, propios de todo proyecto o intención educativa.

Al tratarse de un diseño que nace desde el Enfoque Comunicativo, los tópicos o situaciones comunicativas desencadenarán las funciones gramaticales (qué comunicar de la lengua) y éstos encontrarán, en las habilidades lingüísticas, las herramientas para la construcción comunicativa.

\section{D.1.3.1. Tópicos}

En un modelo comunicativo, un tópico es el asunto sobre el que versará la comunicación y con ello la interacción entre los hablantes.

Al tratarse de un modelo básico que desarrolle habilidades primarias, se pretende que estos tópicos sean generales y, por lo mismo, del interés común del estudiante del Bachillerato.

\section{D.1.3.2. Funciones Gramaticales}

Una función gramatical, de acuerdo con Wilkins, es el uso cotidiano, real que un hablante hace de una lengua. Lo que determina una función es la necesidad de comunicar algo en particular (por ejemplo, saludar a alguien, despedirse, ofrecer una disculpa); lo que dicta el correcto uso de una función es la noción, el deber ser de la lengua.

A partir de una serie de nociones, se articula una función. La función, en síntesis, es la puesta en marcha de la lengua a partir de un mínimo de corrección gramatical ${ }^{18}$.

\footnotetext{
${ }^{18}$ Es frecuente la confusión entre los conceptos de noción y de función. Persiste en el ánimo de muchos alumnos el que en ocasiones ellos quisieran "aprender más gramática". Es lógico, al Enfoque Comunicativo no le interesa la Gramática per se, sino a partir de intenciones y usos comunicativos.
} 


\section{D.1.3.3. Habilidades Lingüísticas}

Una habilidad lingüística es la posibilidad real que el hablante tiene para interactuar con otros o consigo mismo. Es lo que él puede hacer con un sistema de lengua determinado.

El Enfoque Comunicativo establece que son cuatro las habilidades lingüísticas primarias (hablar, escuchar, leer y escribir). Jeremy Harmer señala que las habilidades lingüísticas pueden dividirse en dos grandes categorías ${ }^{19}$ :

1. Habilidades naturales. El autor señala como naturales la capacidad de hablar y de escuchar porque no siguen un proceso de aprendizaje sistemático, ni formal. Se trata más bien de procesos inconscientes de adquisición. En el ámbito de la enseñanza de las lenguas extranjeras, las habilidades naturales (speaking and listening) son herramientas que el estudiante trae consigo ${ }^{20}$.

2. Habilidades aprendidas. Dentro de éstas, encontramos la lectura y la escritura (reading comprehension and written production). Estas habilidades se aprenden en situaciones formales, casi siempre escolarizadas.

Una división distinta es la que hace Penny Ur, quien establece que las habilidades pueden dividirse en $^{21}$ :

- Habilidades de percepción: lectura y comprensión auditiva. Son consideradas como aquellas que demandan un "proceso intelectual".

${ }^{19}$ HARMER, Jeremy (1997). The practice of English Language Teaching. New York: Longman.

${ }^{20}$ El traer consigo estas habilidades por un proceso natural de adquisición puede, muchas veces, viciar los procesos de desarrollo de las habilidades lingüísticas en lenguas extranjeras. Ello se explica por el hecho de que si el estudiante no sabe hablar con propiedad en su lengua materna, será difícil hacerlo con corrección en la lengua extranjera. Lo mismo ocurre con la comprensión auditiva: a veces el estudiante oye, pero no escucha.

${ }^{21}$ UR, Penny (1997). A course in language teaching. Cambridge: Cambridge University Press. 
- Habilidades de producción: oral (speaking) y escrita (writing). Están asociadas estrechamente a las habilidades de percepción. Son la consecuencia natural de las habilidades de producción ${ }^{22}$.

Una vez consideradas ambas categorías, será tarea del docente saber en qué momento y bajo qué circunstancias desarrollará determinadas habilidades lingüísticas en los alumnos.

\section{D.1.4. MECANISMOS DE EVALUACIÓN}

Todo proyecto educativo necesariamente debe evaluarse. Dentro de los mecanismos de evaluación que contempla el diseño curricular, se pretende que éste viva dos momentos:

- Evaluación interna. Un mecanismo de evaluación lo suficientemente representativo para evaluar si los objetivos, contenidos, actividades de aprendizaje y materiales son los pertinentes para producir verdaderamente un desarrollo de habilidades que redunde en aprendizajes reales que se conviertan en auténticas competencias. Recojo la intención de César Coll de realizar al interior del diseño curricular una evaluación que viva tres fases: inicial, formativa y sumativa.

- Evaluación externa. A fin de garantizar la calidad de los procesos educativos de este proyecto educativo, se sugiere la necesidad de ser evaluado por las autoridades correspondientes. Una evaluación externa ayudaría a corregir los procesos que pudieran estar mal fundamentados o no suficientemente desarrollados.

\footnotetext{
${ }^{22}$ Metodológicamente se sugiere que después de una actividad que involucre una habilidad de percepción (escuchar una entrevista, por ejemplo), se pida a los estudiantes que desarrollen una habilidad de producción (manifestar qué escucharon, escribir un comentario...).
} 


\section{E. ESQUEMA INTEGRADOR DE LA PROPUESTA CURRICULAR}

\section{MARCO GENERAL: BACHILLERATO}

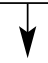

NECESIDADES: ENCONTRAR CORRESPONDENCIA DE OBJETIVOS ENTRE

- COMPETENCIAS ACADÉMICAS BÁSICAS Y

- DESARROLLO DE HABILIDADES LINGÜÍSTICAS BÁSICAS

$\checkmark$

DISEÑO CURRICULAR PARA LA ENSEÑANZA DEL INGLÉS

- OBJETIVOS: Desarrollo de habilidades lingüísticas primarias.

- SELECCIÓN DE CONTENIDOS: Situaciones comunicativas reales.

\section{FUNCIONES:}

Usos de la lengua normados gramaticalmente.

\begin{tabular}{|l|}
\hline HABILIDADES LINGÜÍSTICAS PRIMARIAS: \\
$\bullet$ Hablar \\
- Escuchar \\
$\bullet$ Leer \\
• Escribir
\end{tabular}

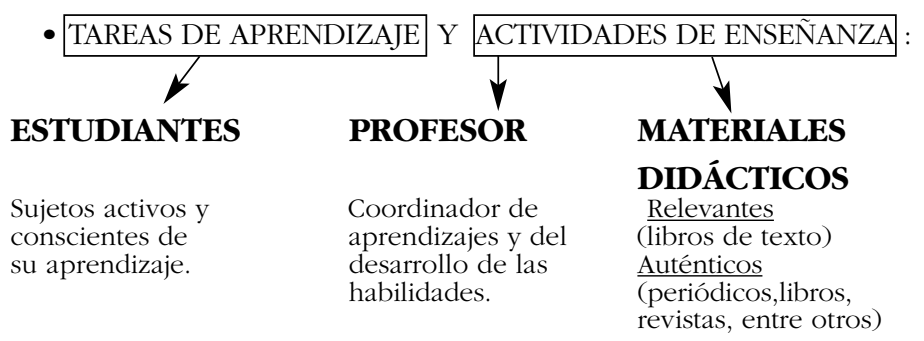

- COMPONENTES CURRICULARES

- Tópicos

- Funciones

- Habilidades lingüísticas

SYLLABUS O

- Evaluación SECUENCIA DE CONTENIDOS 